\title{
Informative titles described article content
}

\author{
Jessie McGowan and Peter Tugwell
}

\begin{abstract}
Objective - To describe the implementation of the new policy of the Journal of Clinical Epidemiology (JCE) to use informative titles for newly submitted articles. Setting - JCE provides timely, authoritative studies developed from the interplay of clinical medicine, epidemiology, and biostatistics. Articles are oriented toward epidemiological methodology, clinical research, or both. Methods - An associate editor is responsible for ensuring that article titles are informative. Authors are instructed to submit titles that are simple declarative statements summarizing the message of the article as succinctly as possible. The informative titles should include the "answer" within the title (the main message of the conclusion), be no longer than 15 words, and state verbs in the past tense for individual studies (whose results might be overruled by later studies or meta-analyses) and in the present tense for systematic reviews (whose results are unlikely to be overruled by later studies). Results - The new criteria were partially implemented in early 2003 with full implementation in 2004. Due to the editorial process, new journal issues with declarative titles started appearing in the fall of 2004. Conclusion - It is hoped that informative titles will help JCE readers to better assess the content of the information in the article.
\end{abstract}

The Journal of Clinical Epidemiology (JCE) is a monthly scholarly journal published by Elsevier Science. The editorial base for the journal is co-located in Ottawa, Canada, and Maastricht, The Netherlands, with two editors and four associate editors. JCE has been in press since 1955. The journal's aims are to promote the quality of clinical epidemiological research and to improve the knowledge base for the diagnosis, prognosis, prevention, and treatment of health conditions through the advancement and application of innovative methods.

JCE continually tries to improve its usefulness to its readers. An editorial decision was made in 2003 to implement the use of an "informative title" or a "declarative title" to help readers distill the content of articles more quickly (the elements of the informative title are shown in Table 1). The new criteria were partially implemented in early 2003, and authors were requested to submit articles with informative titles, as noted in the editorial of the first issue of 2004 [1]. Due to the time involved in the editorial process, new journal issues with informative titles started appearing in the fall of 2004. An associate editor, Jessie McGowan, with assistance from David Sackett, a member of the Policy Advisory Board, was responsible for reviewing the titles of accepted papers.

An informative title gives the conclusion of the article. It was felt that by using more informative titles, readers of JCE would be able to better assess the content of the information in the article. However, there is no evidence to date of the effectiveness of using informative titles. An early reference in the medical literature to the use of informative titles was in 1994, when ACP Journal Club decided to adopt this convention for its titles. They hypothesized that informative titles would help readers decide which abstracts to pay attention to and help busy clinical readers become even more efficient in their efforts to keep up with the literature [2]. Smith suggested using informative titles based on the success of journalists, "who know a thing or two about getting people to read what they write, and use declarative titles and active verbs" [3]. Other hypotheses from the editorial base of JCE included how the use of titles could positively affect the impact factor for JCE. For example, they asked if the use of informative titles could lead to better indexing, or would more understandable titles lead to more referencing of JCE articles by other authors? The answers to these questions are still pending.

There is some controversy about the usefulness of informative titles. Goodman points out that there may be arguments for reviews and editorials carrying informative titles, but they are too often wrong to have any place in the reporting of research [4]. Journals should ask for indicative titles or alter investigators' informative titles during subediting. At the July 2005 editorial meeting of JCE, some concerns over the use of informative titles were raised by a member of the Policy Advisory Board. A decision was made to be less stringent on the use of study architecture in titles where descriptive methods were used and to try to shorten the length of titles. However, overall, the editorial board for JCE is very pleased with the use of informative titles. Anecdotally, the impact factor for JCE increased to 2.654 in 2004 from 2.039 in 2003.

J. McGowan ${ }^{1}$ and P. Tugwell. Institute of Population Health, University of Ottawa - Ottawa Health Research Institute, Room 206, 1 Stewart Street, Ottawa, ON K1N 6N5, Canada.

${ }^{1}$ Corresponding author (e-mail: jmcgowan@uottawa.ca). 
Table 1. Elements of an informative title.

1. Give the results

Should include the 'answer' (the results or main message of the conclusion) within the title

2. Use the past tense for a single investigation or present tense for a systematic review

Should state verbs in the past tense for individual studies (whose results might be overruled by later studies or meta-analyses) and in the present tense for systematic reviews (whose results are unlikely to be overruled by later studies)

3. Name the study architecture

Should include the design within the title, if it is a formal study

4. Should be no longer than 15 words

5. Should not include formal study names

\section{References}

1. Knottnerus A, Tugwlell P. The new look and focus of JCE. $J$ Clin Epidemiol. 2004;57(1):1.

2. Haynes RB. More Informative Titles. ACP J Club. 1994;121(1):A10.
3. Smith R. Informative titles in the BMJ. BMJ. 2000;320:915.

4. Goodman NW. Survey of active verbs in the titles of clinical trial reports. BMJ. 2000;320:914. 